\title{
Online Polling Scheme
}

\author{
K.Sivaraman, A.V.Allin geo, G.Michael, S.Pothumani
}

\begin{abstract}
Online polling system is the web based project to build for voting online among between the people and the citizen.The word "Vote" means to choose form the list, to elect, or to determine. It promotes the casting online voting from any location over the world. Online polling system analysis the real-time implementation or virtual.it helps to reduce thewaste of time, no paper work, no count of votes by hand and no hand-work. It prevents from the chance of falsevoting. It's very simple, safe, secure and take less-time to conduct votes. The main objective of online polling system to allow the people to cast their votes by online over the world .Prevent voters from voting more than once for the single people or person.Easily handled the larger voters andusers. We are developing an on-line voting system by taking advantage of the database with to store a web interface. We have to create the table for store the data into the database. For identification we use the 'keys', which is called the "primary keys "or may be for "forieng key", which should be should be unique or not null.Here,we requiredhardware as well as software component.For developing this polling first we create or the moduls then after we integrate all the module together to build and enhance the project.
\end{abstract}

Keywords:[Homomorphic,encryption,Blindsignature,Security, A SP.Net,Evoting,Registration,CSS,MySQL,Oracle]..

\section{INTRODUCTION}

Failures Online polling systemare some operational work, which is done through the system byusing Hardware and software.Some operation like:-Reading information,checking information which is filled by voters, not accept wrong information. Information available at time and provide security for the data.GUI should must be in only English.It is more convenient to explore with internet explorer.Online voting system should must be contain voters information in the database,voters name should be with unique ID number and also calculate the whole number of votes.It gives more accuracy,Reliabilty,No-Redundancy,Easy to operate. In the case of Hardware requirement use processor,hard-disk and RAM. Similarly, In the case of Software requirement use window XP operating system,ASP.Net framework for the front-end and MS Excel and SQL server to store data-information for the

Revised Manuscript Received on July 22, 2019.

K.Sivaraman, Department of Computer Science and Engineering, Bharath Institute of Higher education and research, Chennai, India

Allin Geo, Department of Computer Science and Engineering, Bharath Institute of Higher education and research, Chennai, India

G.Michael, Department of Computer Science and Engineering, Bharath Institute of Higher education and research, Chennai, India

S.Pothumani, Department of Computer Science and Engineering, Bharath Institute of Higher education and research, Chennai, India
back-end.Easily handled the larger vote and users. There is a database which is maintained by (IEBC) means, the International Electoral and BoudariesCommission .After the registration, the voter is assigned a secret voter ID with which he/she can use to log into the system and enjoy service provide by the system such as voting. In online voting system authentication is the main is problem,only authenticated user is voting for candidate. Only

authorize person can give their vote. Person can be authorizing by some method that can be personal identification number(PIN), the authentication data are collected by the users.All the are verified by main database then allow for that voter.

\section{BACKGROUND WORK}

[1]. Detailed Fujioka[1992], Developed a voting schema using blind signature, it allows the document should be signed with the secret information in the ballot(voting box).In earlier the document placed with the sheet of carbon-paper inside the envelope. When we signature outside of the envelope the automatically comes inside the envelope through the carbon sheet-paper.

[2]. Neumann[1993], Given the idea for "Generaticcriteria"this idea helps to promotes the tempers from the violence.Tempers means,no one will harm your ballots box among between the people.It is extremely difficult to customize.

[3]. Philip[1995], Represent the remote voting scheme apply the technique of blinded signature to voters ballot. It is very difficult for any-one to trace the ballot(box) back to the voters. It desired properties of privacy, universal verifiability, and convenience.

[4]. Crno[1997],proposed and implement a protocol based on fujioka's schema called sensus.Drawbacks ofthe blind signature protocol is the voter has to wait till the voting has ended before the voter can verify the casted vote was corrected once. The implementation of sensus system employs a pollester agent that perform all cryptographic function with program on the voters.

[5]. YahudaLindell [2000], Proposed the comparision of symmetric key and public key crptosystem.discussed about the authentication achievement by using the symmetric key cryptosystem and Asymmetric key crptosystem.Symmetric key encoding algorithm to providing high security in card application when compared to public key algorithm. But Asymmetric encoding provide the function than the symmetric encryption at the expense of speed and hardware 
cost.

[6]. Aviel[2002], The security considerations for remote electronic voting in public election.He examine the feasibility of running national federal elections over the internet. He finally conclude that at present the infrastructure is inadequate for remote internet voting.

[7]. Liaw[2004], Grouping the properties of electronic voting system \& how scheme try to satisfy these properties is also mentioned. Voting should must be uniform and secret , only eligible person must be vote. Voter should must not be able to prove in favourof he/she voted.

[8]. Sonja Hof [2004], Repote about biometric approaches to the electronic voting. Biometric approach may be fingerprint, iris, face, voice, signature, and may be sometime DNA analysis and so on. Biometric approaches is not interact with any-one. It is only access through the voters or users.

[9]. Kalaichelvi[2005], Develop a new technique for encoding or decoding. The substitution and inverse substitution table for encoding and decoding. This table based on ASCII value and the key value.

[10].Sampigethaya[2006], The electronic - voting protocols proposed which is meant to fulfill different requirement using tools and primitives. Primitives are basically blind signature and homographic encoding.

[11]. Chetinkaya[2007], Reporthe verification and validation in e-voting and given the accurate definition for verification and validation. In initially, problem for designing and developing secure - electronic voting system.

[12].Sarah [2008], Report the efficiency of DRE with the traditional machines. In the case of user satisfaction, the DRE was significantly better than older methods but the participants were much more satisfied with experience voting on DRE . It means, the little difference between DRE and traditional method in efficiency.DRE stands for[Direct -recording electronic voting machine].

\section{EXISTING WORK}

- Paper-based visiting system originate as a system where votes are cast and counted by hand.

- With the appearance of electronic tabulation or DRE machine system came where paper card or sheet could be marked by hand counted electronically.

- These system include punch card voting, mark-sense and later digital pen voting system

\section{PROPOSAL WORK}

This project work to find the polling system for vote. It helps to save time and prevent from the error. I will make easier to vote through online. For voting we create home page. The candidates must necessary to fill a from the register, providing voting-details. This entry is checked by the system database to full fill the requirement. After this entry the candidates are easily eligible to vote then user Id and password is given to vote.

The proposed online polling system in ASP.net with following aim:-

a) Count the total numberof vote casting b) Calculate the percentage of total voters.

c) Calculate the total votes cast for each candidate.

d) Calculate the percentage of votes for each candidate.

e) Check duplicate and error in voting.

f) Finding the winning candidates in the election based on the total votes they received.

\section{Architecture:-}

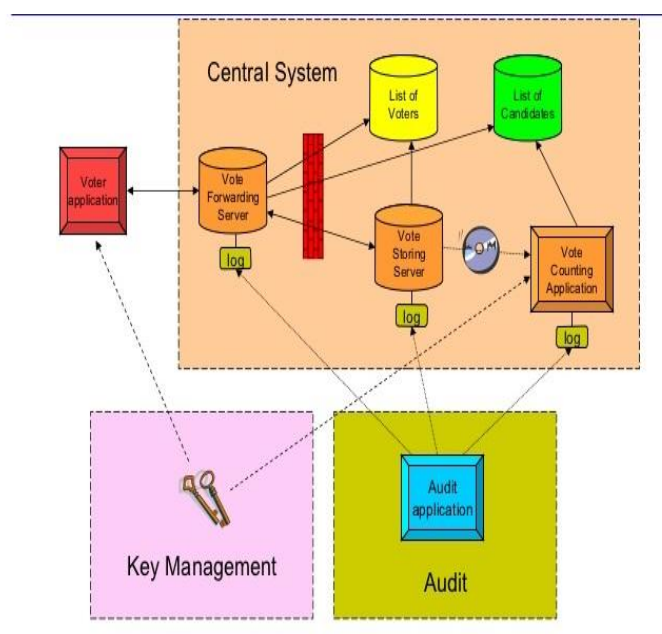

a. Explanation:-

Voter Application:- A voting advise application or Voting Aid application(VAA) is a web application. It helps voters find a candidate or a party that find a candidate or a party that stands closest to their preferences.VAAS are a new phenomenon in modern election campaigning and active towards a goal.

Central System:-Central system is the system that utilized a single source and distribution system for an area.

Central system contains some features :-

$>$ List of voters.

$>$ List of candidates.

$>$ Vote forwarding server.

$>$ Vote storing server.

$>$ Vote counting Application.

List of voters:-A voters list is a list of names and addresses of eligible voters for an upcoming election. The voter's list it is a fundamental component of any voting system. The purpose of the voters list is to conform the eligibility of the voters to participate .There is the condition to eligible for casting vote which is those candidate having age should be 18 years old or more than 18. List of candidates:-The condidate list is defined as a person who is seen as suitable for a position who is taking a test for acceptance. Which has been choosen by a political party to run for office.

Vote forwarding server:- Vote forwarding server is the server when we give input the vote for casting then it will forwarding to vote storing server then this phenomenon of processing is known as vote forwarding server.Access the for both list of the voter nd the candidate list. 
Vote storing server:-storing server is use to store the data of the list of voter for conducting votes.It is connected through the SQL serever to retrieve the data from the database.

Vote counting Application:- Counting application is use for count the votes of the voters by using application.It declare the final result of the votes after the counting of the voter.It is handled by thekey-management and Audit.

Key Management :-Key-management system works in conjuction, which provides extensive reporting options centralized programming, advanced network capabilities and real-time transaction polling. Accessible by multiple administrators from remote locations via standard internet connection.

\section{Some features of Key-management:-}

$>$ User-friendly Interface.

$>$ Real-time Transaction polling.

$>$ Maximized Network capabilities.

$>$ Real-Time Reporting.

Audit:-An audit is an objective examination and evaluation of the financial statements of an organization to make sure that the records are a fair and accurate representation of the transaction the claim to represent.It can be done internally by voters of the organization. A post-election audit checks that the equipment and procedure used to court votes during an election worked properly, and that the election yielded the correct result.

\section{Benefits of Audits:-}

* Revealing when recounts are necessary to verify election outcomes.

* Contol the fraud.

* Providing for continuous improvement in conduct of elections.

* Promoting public confidence in elections.

Collaboration of voting system :- In Administrator used to maintain (add update modify and delete and detail of voter and candidates.

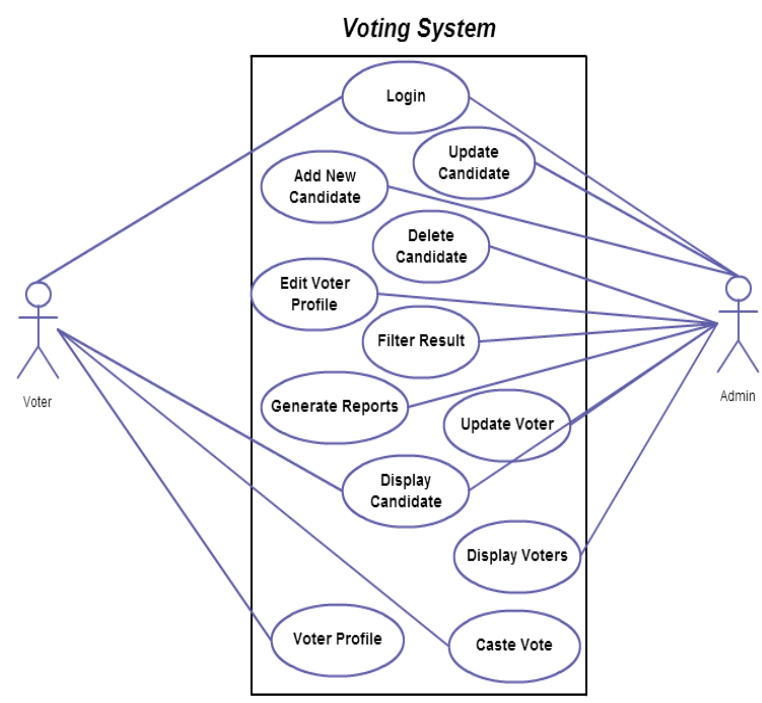

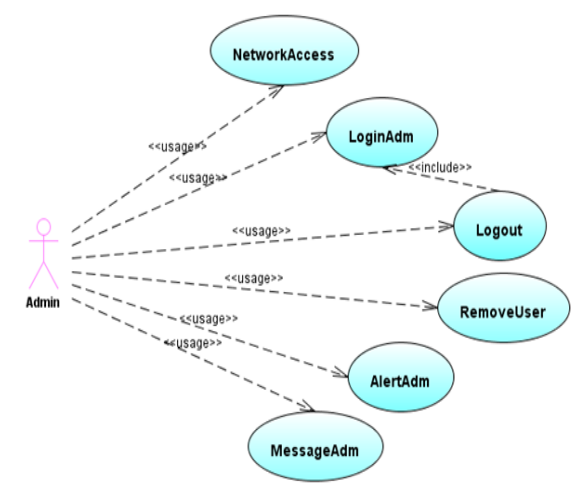

\section{CASE STUDY}

The case study undertaken was planned to be executed on Tomcat as it is commonly used for business purpose, it is Java technology based and functionality of Apache web server is a subset functionality of this. Similar approach should hold good for $\mathrm{C \#}$ as well. The windows environment hosted all these components and the results were tested using manual injection of faults in selective classes. The environment used JDK 7 along with Apache Tomcat 7.0.x and $\log 4 \mathrm{j}$. Eclipse 4.4 Luna was used as the development and build environment. Thebootstrap process (the server start) has been taken to study the case. graph was generated for the bootstrap classes and logging code has been added. The added code was reviewed and any tool related errors were corrected. The code was executed and logs are saved.

\section{CONCLUSION}

The system is design which is based on the technology we can say as the smart voting system using photo or relational data-baserecognisation. It also promotes the counting of votes and required minimum number of officers. Result is quickly transfer to the Data-base. Here,we implement an online voting system by which the user can vote over the online. The great advantages for NRI and person who is outside of the country ,they can also vote through online by internet. This project isvery flexible and economically improvement features and sub-module can added as per the requirement.Voter will not be needed to go to voting booth.They can vote from anywhere It will also enables voters to register themselves online. Problem of fake voting is also eliminated.

\section{REFERENCES}

1. P. Paillier, Public-Key Cryptosystems supported Composite Degree Residuosity categories, Eurocrypt '99 http://www.euractiv.com/en/egovernment/estonia-country-world-introd uce-internet-voting/article-145735, Esthonia 1st country within the world to introduce web pick, October 2005.

2. A.J. Menezes, P. C. van Oorschot, and S.A. Vanstone, vade mecum of Applied Cryptography, CRC Press, 1997. 
3. Sampigethaya ,Threshold Cryptography internet Service User's Guide, University of Colorado - city Master's Project, 2006.

4. AnkitAnand, PallaviDivya: associate degree economical on-line electoral system in International Journal of contemporary Engineering analysis (IJMER) Vol.2, Issue.4, July-Aug. 2012 pp-2631-2634.

5. S. W. Ambler, method Patterns: Building massive Scale Systems exploitation Object Technology,Cambridge University Press, 1998.

6. M. Andrews and J. A. Whittaker, a way to Break internet Software: useful and Security Testing of internet Applications and internet Servers. Addiso,Wesley, 2006.

7. Sarah, AlmytaSystems, purpose of Sale Systems http://systems.almyta.com/Point_of_Sale_Software.asp. Accessed on twentieth October 2008.

8. Alexander. Stakeholders: WHO is your system for IEEE: Computing and management Engineering,26 April 2003.

9. Kalaichelvi,Chetinkaya. Face Spoof Detection with Image Distortion Analysisl, IEEE Transactions on data Forensics and Security, 2005.

10. A Survey of Current Secret-Ballot Systems", David. Chaum.

11. Fujioka , Neumann. A practicalsecret votingscheme for large scale elections. In Workshop on the applying of science Techniques: Advances in branch of knowledge, ASIACRYPT '92, pages 244-251. Neumann, 1993.

\section{AUTHORS}

\section{PROFILE}

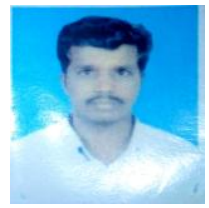

Mr.K.Sivaraman, Assistant

Professor, Department of

Computer Science \&

Engineering, Bharath

Institute of Higher Education

and Research, Chennai,

India

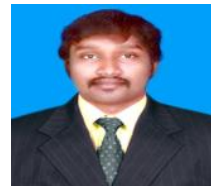

Mr.A.V.Allin Geo, Assistant

Professor, Department of

Computer Science \& Engineering,

Bharath Institute of Higher

Education and Research, Chennai,

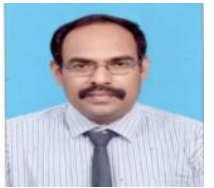

Mr.G.Micheal,

Associate

Professor, Department of Computer Science \& Engineering, Bharath Institute of Higher Education and Research, Chennai, India

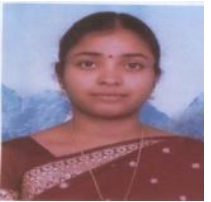

Mrs.S.Pothumani, Assistant Professor, Department of Computer Science \& Engineering, Bharath Institute of Higher Education and Research, Chennai, 